\title{
Carole Ho
}

More than $98 \%$ of the Alzheimer disease candidates that make it into phase III trials subsequently fail, according to a 2014 review of the field. Failure rates for Parkinson disease and amyotrophic lateral sclerosis (ALS) drug candidates are similarly dire. And yet, the 1.5-year-old biotechnology company Denali Therapeutics has already raised a massive US $\$ 349$ million to tackle these and other neurodegenerative diseases. The company is about to advance its first candidate into the clinic, and Chief Medical Officer Carole Ho, former vice-president of early clinical development at Genentech, is making plans. She tells Asher Mullard that genetic insights into the biology of neurodegenerative disease could provide new therapeutics within 10 years.

\section{Q What is Denali's overall science strategy?}

There is increasing hope in neurodegenerative diseases because of the growing understanding of the biology of these diseases. Around 5 years ago, really the only targets for Alzheimer disease were in the amyloid beta pathway. It wasn't that companies weren't interested in other targets; it just wasn't clear what those targets would be. Now, genetic findings, in combination with greater understanding about how the disease unfolds, have really opened the door to other therapeutic targets. The same is true of Parkinson disease and amyotrophic lateral sclerosis (ALS).

In therapeutic areas where genetic discoveries have provided insight into the biology of disease, therapeutics often followed around 10 years later. This is not just the case in oncology. In hypercholesterolaemia, researchers identified proprotein convertase subtilisin/kexin type 9 (PCSK9) mutations in 2003, and by 2015, the first PCSK9-targeting therapeutics were approved.

The genetic risk factors that have been linked to neurodegenerative diseases to date include our first set of targets: receptorinteracting serine/threonine-protein kinase 1 (RIP1), apolipoprotein E4 (APOE4) and leucine-rich repeat serine/threonine-protein kinase 2 (LRRK2). Our belief is that we will start to see therapeutic agents that are effective in the clinic in this decade.

Another genetic risk in Alzheimer disease that we've focused our attention on is microglia - support cells that are responsible for inflammation and homeostasis in the nervous system. In genome-wide association studies (GWAS) in Alzheimer disease, $50 \%$ of the hits are in microglial genes.
Q We've understood the genetics of sickle cell disease for 60 years, and have not yet translated this into therapeutics. Is your hope that genetics could open up neurodegenerative diseases within only 10 years over-optimistic? You need to be optimistic in this business. But my sense is that even emerging data from the anti-amyloid therapies are starting to show hints of efficacy. And as we learn more about the genetic risks of these diseases, we'll start to understand how we can use combination therapies to hit multiple pathways at once, which may be what is required to see significant benefits.

Q. How does your focus on the genetics of neurodegeneration affect your clinical strategy? One thing that is important in our overall strategy is our ability to identify the individuals who are most likely to respond to our drugs, maximizing our chances of success in the clinic. The genetics in some cases helps us to understand what this patient population might look like. In Parkinson disease, patients with LRRK2 mutations are more likely to respond to a LRRK2 inhibitor. For other targets, it is not as straightforward. We are looking at how to measure inflammation in the brain, for example, as a means of identifying individuals for clinical trials in other programmes.

Another area that we are investing in is better end points. We are collaborating with ALS Therapy Development Institute (ALS TDI) on their Actigraph technology, a wearable accelerometer that may help us to assess the clinical decline of ALS patients. We are interested in understanding how changes in function, as assessed using the Actigraph, relate to approvable end points. At this point we are focused on this not so

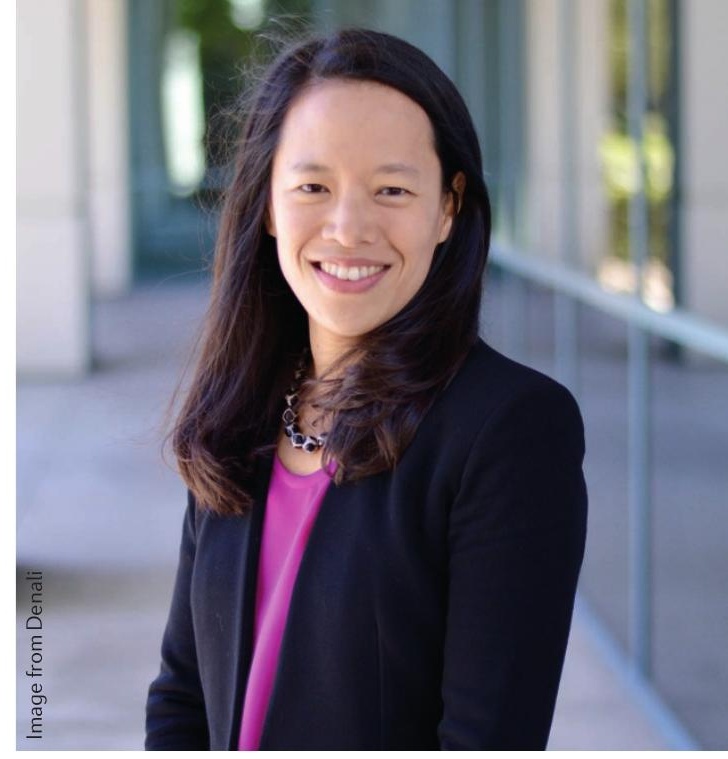

much as a registrational end point, but rather as a means of finding more sensitive ways of dose finding and detecting efficacy in early-stage studies.

I can't overemphasize the importance of this. When you look at neurodegeneration drug development, the majority of studies never adequately looked at whether a drug was getting into the brain, was hitting its targets and was producing a clinical signal beyond the registrational approval end points.

\section{Q How is your strategy different from large} pharma and other biotechs?

Neurodegeneration is a high-risk area. What we've seen with large pharmaceutical companies, which have diversified portfolios of projects across various indications, is that there is a prioritization process that looks at the cost of developing a therapeutic agent and the likelihood of success. This makes sense for their business model. And when you look at therapeutic areas where the genetics and the science are more advanced - such as in oncology - there is a higher likelihood of success in these therapeutic areas and so they are highly prioritized. Neuroscience indications and neurodegeneration are, by contrast, not fully prioritized.

Traditional start-ups, on the other hand, often only have enough capital to bring one drug to the market, and have an incentive to keep developing one drug for as long as possible. Sometimes a critical experiment may not be done at those companies because if it fails, that's the end of the company.

When Denali was conceived, the founders wanted to raise enough capital to succeed in neurology by being able to develop several molecules in parallel. Neurodegenerative drug development is not for the faint of heart. We expect that there will be many failures. But we can fail quickly and move to the next thing. We have enough funding that we can focus on the critical experiments, and make decisions for our candidates based on the data. 